\title{
FLAT BUNDLES WITH SOLVABLE HOLONOMY. II. OBSTRUCTION THEORY
}

\author{
WILLIAM M. GOLDMAN
}

\begin{abstract}
Necessary and sufficient conditions for a connected solvable Lie group $G$ are given so that every flat principal $G$-bundle over a $\mathbf{C W}$-complex is trivial after passing to a finite covering space.
\end{abstract}

In [2] it was proved that if $\xi$ is a flat principal $G$-bundle over a $C W$-complex $M$ where $G$ is a solvable linear Lie group then there is a finite-sheeted (Galois) covering $p: \hat{M} \rightarrow M$ such that $p^{*} \xi$ is trivial. (If such a covering $p$ exists we say that $\xi$ is virtually trivial.) In this note we extend this result to general solvable Lie groups $G$, giving necessary and sufficient conditions for the above conclusion. In particular we show that there exist flat principal $G$-bundles which are not virtually trivial.

TheOREM. Let $G$ be a connected solvable Lie group. Then the following conditions are equivalent:

(a) The commutator subgroup $G^{\prime}$ of $G$ is simply connected (in its Lie group topology);

(b) every flat principal $G$-bundle over a $C W$-complex is virtually trivial.

Proof. (a) $\Rightarrow(\mathrm{b})$. Suppose $G^{\prime}$ is simply connected and $\xi$ is a flat principal $G$-bundle over $M$. We shall show that all the obstructions to trivializing $\xi$ vanish after pulling back over a finite covering. (See Steenrod [7] for a description of the method of obstruction theory.) The first obstruction lies in $H^{1}\left(M ; \pi_{0}(G)\right)$ and therefore vanishes since $G$ is connected. The next obstruction (which we denote $\left.o_{2}(\xi)\right)$ lies in $H^{2}\left(M ; \pi_{1}(G)\right)$. Let $\pi=\pi_{1}(M)$ and let $\phi: \pi \rightarrow G$ be the holonomy homomorphism of $\xi$. Then the obstruction $o_{2}(\xi)$ is simply the obstruction to lifting $\phi$ to a homomorphism $\pi \rightarrow \tilde{G}$, where $\tilde{G}$ denotes the universal cover of $G$. Although in general $o_{2}(\xi)$ does not vanish, we will show that it is a torsion class.

We begin by showing that for every homology class $z \in H_{2}(M ; Z)$, the product $o_{2}(\xi) \cdot z \in \pi_{1}(G)$ is zero. Since $\xi$ is a flat bundle, its classifying map factors through the Eilenberg-Mac Lane space of type $K(\pi, 1)$. Indeed its classifying map may be taken to be the composition $M \rightarrow^{f} K(\pi, 1) \rightarrow^{B \phi} K(G, 1)$ where $f$ is a map inducing an isomorphism on fundamental groups and $B \phi$ is the map induced by $\phi$. Thus $\xi$ is induced by a flat principal $G$-bundle over $K(\pi, 1)$ and we may assume with no loss of generality that $M$ is a space of type $K(\pi, 1)$.

Received by the editors October 21, 1980.

1980 Mathematics Subject Classification. Primary 22E25, 55R10; Secondary 55R15.

Key words and phrases. Solvable Lie group, flat bundle, obstruction, fundamental group of a surface, finite covering space. 
In Hopf [4] the following description of $H_{2}(M ; Z)$ is given when $M$ is a $K(\pi, 1)$. Write $\pi$ as a quotient $F / R$ where $F$ is a free group on some generators $f_{1}, \ldots, f_{k}$ and $R$ is a normal subgroup of $F$. Then $H_{2}(M ; Z)$ is $(R \cap[F, F]) /[F, R]$ where $[A, B]$ denotes the group generated by all commutators $[a, b]=a^{-1} b^{-1} a b$ where $a \in A, b \in B$. The above formula is proved by showing that each homology class $z \in H_{2}(M ; Z)$ is represented by a map of a closed orientable surface $F$ into $M$; such maps are represented (up to homotopy) by homomorphisms $\pi_{1}(F) \rightarrow \pi$. Using the well-known presentation of the fundamental group of a surface $\pi_{1}(F)=$ $\left\langle a_{1}, b_{1}, \ldots, a_{n}, b_{n} \mid\left[a_{1}, b_{1}\right] \cdots\left[a_{n}, b_{n}\right]=1\right\rangle$ one sees that homology classes $z$ correspond to relations in $\pi$ of the form $\left[A_{1}, B_{1}\right] \cdots\left[A_{n}, B_{n}\right]=1$ where the $A_{i}, B_{i}$ are themselves words in the generators of $\pi$.

Let $R=R\left(g_{1}, \ldots, g_{n}\right)$ be a relation in the generators $g_{1}, \ldots, g_{n}$ of $\pi$ such that under the above correspondence $R$ corresponds to the homology class $z$. Then $o_{2}(\xi) \cdot z$ may be computed as follows. Choose lifts $\phi\left(g_{i}\right)^{\sim}$ of the $\phi\left(g_{i}\right)$ to $\tilde{G}$; then consider the element $R\left(\phi\left(g_{1}\right)^{\sim}, \ldots, \phi\left(g_{n}\right)^{\sim}\right)$ of $\tilde{G}$. Since it is a product of commutators and choices of lifts $\phi\left(g_{i}\right)^{\sim}$ differ by elements of the center of $\tilde{G}$, this element is independent of the choice of lifts. Moreover since $R\left(g_{1}, \ldots, g_{n}\right)=1$, the constructed element of $\tilde{G}$ lies in the kernel of $\tilde{G} \rightarrow G$ which we identify with $\pi_{1}(G)$. It is not difficult to verify (see Milnor [5] and Hirzebruch [8, §I]) that $R\left(\phi\left(g_{1}\right)^{\sim}, \ldots, \phi\left(g_{n}\right)^{\sim}\right) \in \pi_{1}(G)$ equals $o_{2}(\xi) \cdot z$.

Now $R$ is a product of commutators, so $o_{2}(\xi) \cdot z$ lies in the commutator subgroup $(\tilde{G})^{\prime}$ of $\tilde{G}$. On the other hand it lies in $\pi_{1}(G) \subset \tilde{G}$. But $\pi_{1}(G) \cap(\tilde{G})^{\prime}$ is the fundamental group of $G^{\prime}$, so that $o_{2}(\xi) \cdot z=0$ for all $z \in H_{2}(M ; Z)$.

Recall the universal coefficient exact sequence

$$
0 \rightarrow \operatorname{Ext}\left(H_{1}(M ; Z), \pi_{1}(G)\right) \rightarrow H^{2}\left(M ; \pi_{1}(G)\right) \rightarrow \operatorname{Hom}\left(H_{2}(M ; Z), \pi_{1}(G)\right) \rightarrow 0
$$

We have proved that the image of $o_{2}(\xi)$ in $\operatorname{Hom}\left(H_{2}(M ; Z), \pi_{1}(G)\right)$ is zero; by exactness $o_{2}(\xi)$ lies in the image of $\operatorname{Ext}\left(H_{1}(M ; Z), \pi_{1}(G)\right)$ which is a finite abelian group. It follows that $o_{2}(\xi)$ is a torsion class.

To see that $\xi$ is virtually trivial, let $T$ denote the torsion part of $H_{1}(M ; Z)$ and let $p: \hat{M} \rightarrow M$ be the covering space of $M$ corresponding to the kernel of the natural surjective homomorphism $\pi_{1}(M) \rightarrow T$. Since $T$ is a finite group, $p$ is a finite-sheeted covering space. Working through the definitions one sees that the obstruction class $o_{2}\left(p^{*} \xi\right)$ is the image of $k \cdot o_{2}(\xi)$ under $p^{*}: H^{2}\left(M ; \pi_{1}(G)\right) \rightarrow H^{2}\left(\hat{M} ; \pi_{1}(G)\right)$ where $k$ equals the order of $T$. Since $k \cdot o_{2}(\xi)=0$, it follows that $o_{2}\left(p^{*} \xi\right)=0$.

The next obstructions for trivializing $p^{*} \xi$ all lie in cohomology groups $H^{i+1}\left(\hat{M} ; \pi_{i}(G)\right)$ for $i \geqslant 2$. As $G$ is a solvable Lie group, $\pi_{i}(G)=0$ so all the successive obstructions for $p^{*} \xi$ vanish. Therefore we have proved that $p^{*} \xi$ is trivial.

(b) $\Rightarrow$ (a). Suppose that $G^{\prime}$ is not simply connected; we will find a principal $G$-bundle over a surface which is not virtually trivial. Since $G^{\prime}$ is not simply connected, we may find a nontrivial element $c \in(\tilde{G})^{\prime} \cap \pi_{1}(G) \subset \tilde{G}$. Thus there exist elements $\tilde{a}_{1}, \ldots, \tilde{a}_{n}, \tilde{b}_{1}, \ldots, \tilde{b}_{n} \in \tilde{G}$ such that $\left[\tilde{a}_{1}, \tilde{b}_{1}\right] \cdots\left[\tilde{a}_{n}, \tilde{b}_{n}\right]=c$. Denote their images in $G$ by $a_{1}, \ldots, a_{n}, b_{1}, \ldots, b_{n}$; then we may construct a 
homomorphism $\phi: \pi_{1}(F) \rightarrow G$, where $F$ is a closed orientable surface of genus $n$, by sending the usual system of generators for $\pi_{1}(F)$ to the $a_{i}, b_{i}$. We claim the associated flat principal $G$-bundle $\xi$ over $F$ is not virtually trivial. The obstruction to trivializing $\xi$ is the element corresponding to $c$ in $H^{2}\left(F ; \pi_{1}(G)\right) \simeq \pi_{1}(G)$ and is nonzero since the map on fundamental groups induced by the inclusion $G^{\prime} \rightarrow G$ is injective. Moreover if $p: \hat{F} \rightarrow F$ is a $k$-fold covering then the obstructions satisfy $o_{2}\left(p^{*} \xi\right)=p^{*} o_{2}(\xi)$. There is a commutative diagram

$$
\begin{array}{ccc}
H^{2}\left(F ; \pi_{1}(G)\right) & \simeq & \pi_{1}(G) \\
\downarrow p^{*} & & \\
H^{2}\left(\hat{F} ; \pi_{1}(G)\right) & \simeq & \pi_{1}(G)
\end{array}
$$

Since $\pi_{1}(G)$ is free abelian, it follows that $o_{2}\left(p^{*} \xi\right)$ is nonzero. Hence $\xi$ is not virtually trivial. Q.E.D.

REMARKs. (1) For an application of obstruction theory for the (somewhat analogous) case of $G$ locally isomorphic to $\operatorname{PSL}(2, R)$ see $[1, \S 3]$. The first examples of flat $G$-bundles which are not virtually trivial are due to Milnor [5], where $G=S L(2 ; R)$.

(2) If $G$ is a connected solvable Lie group and $G^{\prime}$ is simply connected, then in general it is necessary to pass to a finite covering to trivialize a flat principal $G$-bundle. Here is a simple example. Let $M=R P^{2}$ and $G=S O(2)$. There are two equivalence classes of flat principal $G$-bundles over $M$ : the trivial bundle with total space $R P^{2} \times S O(2)$, and the bundle $\xi$ induced by a faithful representation $Z / 2 \rightarrow$ $S O(2)$. The total space of $\xi$ is easily seen to be homeomorphic to $S^{2} \times S O(2)$. Clearly these two spaces are distinct, so that $\xi$ is nontrivial.

However, the above proof shows that if $H_{1}(M ; Z)$ is free abelian, then every flat principal $G$-bundle over $M$ is trivial.

(3) In Sullivan [6] it is shown that if $G$ is an algebraic group, then there are only a finite number of isomorphism classes of principal $G$-bundles over a base space $M$ as above which admit flat structures. For some $G$ not algebraic counterexamples may be constructed along the lines of $(b) \Rightarrow(a)$ above. Here is the simplest example. Let $\tilde{G}$ be the Heisenberg group consisting of real upper unitriangular $3 \times 3$ matrices, and let $C$ be the cyclic group generated by any nontrivial $c \in$ $\operatorname{center}(\tilde{G})=(\tilde{G})^{\prime}$. Let $G=\tilde{G} / C$. For every $n \in Z$ there exist $\tilde{a}, \tilde{b} \in \tilde{G}$ with $[\tilde{a}, \tilde{b}]=c^{n}$. The images of $\tilde{a}, \tilde{b}$ in $G$ commute so they determine a homomorphism $Z+Z \rightarrow G$. The associated flat principal $G$-bundle over the 2-torus is fiber homotopy equivalent to a circle bundle with Euler number $n$. Thus infinitely many principal bundles occur.

We may interpret these examples in terms of the theory developed by Gromov [3]. In [3] it is shown that for algebraic groups $G$, the image of the natural homomorphism $H^{*}(B G) \rightarrow H^{*}\left(B G^{\delta}\right)$ consists of bounded cohomology classes. (Here $G^{\delta}$ denotes $G$ with the discrete topology.) It follows from the preceding examples that for some nilpotent nonalgebraic Lie groups $G$, the image of $H^{*}(B G)$ $\rightarrow H^{*}\left(B G^{\delta}\right)$ consists entirely of unbounded cohomology classes. 


\section{REFERENCES}

1. W. Goldman, Discontinuous groups and the Euler class, Doctoral dissertation, University of California, Berkeley, 1980.

2. W. Goldman and M. Hirsch, Flat bundles with solvable holonomy, Proc. Amer. Math. Soc. (to appear).

3. M. Gromov, Volume and bounded cohomology, Publ. Math. Inst. Haut. Études Sci. (to appear).

4. H. Hopf, Fundamentalgruppe und zweite Bettische Gruppe, Comment. Math. Helv. 14 (1942), 257-309.

5. J. Milnor, On the existence of a connection with curvature zero, Comment. Math. Helv. 32 (1958), 215-223.

6. D. Sullivan, A generalization of Milnor's inequality concerning affine foliations and affine manifolds, Comment. Math. Helv. 51 (1976), 183-199.

7. N. Steenrod, The topology of fibre bundles, Princeton Univ. Press, Princeton, N. J., 1951.

8. F. Hirzebruch, Topological methods in algebraic geometry, Springer-Verlag, Berlin and New York.

Department of Mathematics, University of Colorado, Boulder, Colorado 80309

Current address: Department of Mathematics, Massachusetts Institute of Technology, Cambridge, Massachusetts 02139 